\title{
Del silenciu al esporpolle identitariu: hacia una normalización de calter «rellacional» del asturianu y del gallegoasturianu na sociedá rizomática d'anguaño / From silence to the strengthening of identities: towards a «relationship» planning of Asturian and Galician-Asturian languages in the current rhizomatic society
}

\author{
GONZALO LLAMEDO PANDIELLA \\ UNIVERSIDÁ D’UVIÉU
}

\begin{abstract}
RESUME: Un saltu de paradigma esixe delles respuestes nueves. La sociedá posmoderna del consumu muévese con inseguridá nuna realidá cambiante, caótica y cada vuelta más rizomática. Búscase l'abellugu d'un bienestar inmediatu pero efímeru, que nunca enllena dafechu. Per otru llau, resulten inda más evidentes la heteroxeineidá cultural del mundu d'agora y les preocupaciones identitaries, motivu pol que recuperen la voz nestos momentos los discursos minorizaos. L'oxetu d'esti trabayu ye plantegar una reflexón teórica, dende'l puntu de vista de la socioloxía del llinguaxe. Propónse asina un analís de la concepción de dos realidaes tan «sólides» como son los procesos de normativización y normalización del asturianu y del gallegoasturianu, dientro d'un marcu de rizoma propiu de la «modernidá líquida» de Bauman. En plenu procesu de normalización social d'estes llingües, tórnase imprescindible deprender a convivir colos esquemes de vida posmoderna y entendelos bien, pa poder trabayar en conxuntu na proyección d'un llabor de normalización granible. Ufiértense, a esti respectu, delles estratexes inspiraes nos modelos rellacionales de desing thinking -propios del marketing one to one- y de les esperiencies de pensamientu «lean», col envís d'estrechar el contactu coles respectives comunidaes de falantes, pa qu'estes sientan les sos llingües afayadices, entiendan la situación actual de diglosia y contribuyan a desaniciala cola so llealtá llingüística.
\end{abstract}

Pallabres clave: llingua asturiana, gallegoasturianu, procesu de normalización, rizoma, modernidá líquida.

ABSTRACT: The emergence of a paradigm shift demands new answers. The Postmodern consumerist society moves with insecurity around this changing, chaotic and increasingly rhizomatic reality. An immediate but ephemeral well-being is always required but it never works enough. On the other hand, cultural diversity and identity concerns are now still more evident. For this reason, a minorized discourse is nowadays recovering its voice and importance. The current paper presents a theoretical framework built on the basis of the sociology of language. The article analyses the conception of Asturian and Galician-Asturian «solid» language planning 
processes within Bauman's «liquid modernity». Understanding and learning to coexist with Post-modern life patterns is essential to reach an effective language planning. To that end, design thinking strategies inspired on one-to-one relational marketing patterns and lean thinking experience will be tackled. These communities of speakers are aimed to feel comfortable in these languages, to comprehend the diglossic situation and to contribute to its abandonment through their language loyalty.

Key Words: Asturian language, Galician-Asturian language, language planning, rhizome, liquid modernity.

\section{ENTAMU}

La nuesa comunidá llingüística lleva sieglos reflexonando sobre les llingües asturiana y gallegoasturiana, desarrollando adulces una conciencia llingüística dende la Edá Media (García Arias 2013) y preocupándose más tarde poles sos característiques internes, pol so caltenimientu nel espaciu y nel tiempu y pola percepción social o identitaria que d'elles se tien. Nesti sentíu, como recueye'l llingüista y escritor Xulio Viejo, dempués d'una etapa moderna de referencies escrites y d'averamientos distintos a estes llingües con una visión de calter más bien filolóxicu o sociollingüísticu, con especial procuru pola dialectoloxía, nes últimes décades surden nuevos trabayos dende la socioloxía del llinguaxe (Viejo 2017), empobinaos a analizar los contestos qu'inflúin nes actitúes de los individuos pa coles llingües.

En paralelo a estos cambeos na conciencia llingüística histórica, la sociedá asturiana foi tresformándose muncho, afayándose na realidá posmoderna occidental d'agora. Por esti motivu -y más cuando nos alcontramos en plenu procesu de normalización llingüística ${ }^{1}-$ tien cabida un analís más sociolóxicu, que pue ampliar la visión del contestu social col que conviven les comunidaes llingüístiques, na busca de nueves orientaciones que permitan avanzar nel procesu de normalización del asturianu y del gallegoasturianu, nun «interesante momentu de reflexón» (Guardado Díez 2008: 13).

Siguiendo esa llinia, cabe entrugase: al falar de les llingües minorizaes d'Asturies, ¿ta atendiéndose lo suficiente a la tipoloxía de sociedá qu'esiste

1 Define'l llingüista Ramón d'Andrés la normalización llingüística como'l «conxuntu sistemáticu d'acciones sociopolítiques que remiten, nel ámbitu llingüísticu, a la consecución d'una normalidá na convivencia de dos o más llingües nel mesmu espaciu social, y más específicamente d'una normalidá pa la llingua o llingües que sufren una minorización» (D’Andrés 2018: 19) y, dientro d'ella, dánse dos planos: normalización social, esto ye, del estatus, y normalización del corpus o normativización. 
güei? ¿Concuerden les estratexes de normalización colos modelos rellacionales de los patrones de consumu que conducen la sociedá posmoderna d'anguaño?

Cola intención de trazar llinies de fuga qu'abran nueves posibilidaes d'investigación y qu'ufierten respuestes a estes cuestiones, preséntase a continuación un analís teóricu y descriptivu centráu na necesidá de comprender los esquemes de la vida posmoderna y de deprender a convivir con ellos, pa proyectar un llabor de normalización más granible que reafirme'l contactu colos falantes. L'analís consta de cuatro puntos principales: en primer llugar, rescátense les nociones de «rizoma» y de «sociedá líquida», nun averamientu al panorama social occidental d'anguaño; en segundu llugar, atiéndese al surdimientu de nueves estratexes de marketing y de modelos de consumu personalizaos a lo cabero del sieglu XX, motiváu pola intensificación del procesu de mercantilización; en tercer llugar, concédese un espaciu al puxu qu'algamen les minoríes dientro d'esti modelu social rellacional; y, como última cuestión, apúrrense exemplos sobre les oportunidaes que puen alcontrar les llingües gallegoasturiana y asturiana coles estratexes que siguen los citaos modelos de «marketing rellacional», p'algamar la normalización social de manera más rápida y efectiva.

\section{LA DÓMINA DEL RIZOMA}

A lo cabero del añu 2018 había yá nel mundu 7.594 millones d'individuos (Grupo Banco Mundial 2019), un númberu creciente nun espaciu xeográficu que nun se consigue estirar. Munchos individuos, toos ellos creadores y víctimes de múltiples circunstancies, suxetos a tresformaciones continues. Esti escenariu constitúi la dómina del «rizoma».

¿Y qué s'entiende por «rizoma»? Pártese d'un conceptu emprestáu de la botánica, disciplina científica que lu define como un tallu horizontal y soterrañu de dalgunes plantes, que supón pa elles un finxu. D'esti tallu espunten les raíces, xabaces, d'un mou imprevisible, raigaños de los que puen espolletar otros rizomes (Alvarado 2008: 93). El rizoma espárdese fuerte y llibre (Deleuze \& Guattari 1977: 16), orixina ñudos y alquier formes y aspectos diferentes. Asina vive.

Los filósofos Deleuze \& Guattari aplicaron el conceptu de rizoma a la filosofía pa falar d'un modelu organizativu ensin orde xerárquicu, onde reina'l caos y los constituyentes puen rellacionase al marxe del llugar qu'ocupen o de les circunstancies que los determinen. Dende entós, son munches les estayes de conocimientu que recuperen la imaxe del rizoma ${ }^{2}$, pa caracterizar les

2 Por exemplu, nel ámbitu de la comunicación dixital (Zapata Duque 2005; López Gil \& Angulo Rasco 2015) o na pedagoxía (Cormier 2008 \& 2014; Maioz Basterretxea 2015). 
estructures complexes d'anguaño, basaes na conexón y na heteroxeineidá.

La sociedá posmoderna ${ }^{3}$ resultante del procesu de globalización abandona la estructura y pasa a configurar tamién una rede rellacional en constante fluxu, una matriz de conexones inciertes (Bauman 2007: 9). Asina, por exemplu, una decisión llocal pue crear una serie d'efectos dominó n'otres partes conectaes de la rede. Son tantes les raíces d'esti tallu de cuasi ocho mil millones d'habitantes y tantes les acciones individuales con repercusión nel conxuntu, que non siempre se lleguen a prever y a controlar esos efectos. Anque les xerarquíes y los poderes entá esisten, nun puen intervenir na totalidá de variables. D'esti mou, el procesu de globalización provoca un cambéu de paradigma, del que deriva una emancipación progresiva del capitalismu d'eses estructures de poder (Bauman 1999a: 199). Nesti sentíu, si nun lo somos yá, vamos camín de convertinos nuna sociedá rizomática.

Alcordies con esti puntu de vista, el sociólogu, filósofu y ensayista Zygmunt Bauman dedicó delles décades a desplicar el panorama social d'anguaño a traviés de fluxos rellacionales, acuñando la espresión «modernidá líquida», pa describir el pasu d'una fase industrial d'organización sistemática, más inflexible y de compromisu mutuu, nomada «sociedá sólida», a un modelu de vida «líquidu» que ruempe con estos esquemes y que nun ye a axustase a nengún otru, porque agora lo duradero y el compromisu espanten (Bauman 2007: 7). La sociedá global ye güei la sociedá del riesgu, de la dulda (Zufiaurre 2007: 15), de l'ambivalencia (Bauman \& Donskis 2013/2015: 13), de la tensión, de les espectatives y de les presiones constantes que se manifiesten como resultáu emocional d'un escesu (Simmel 2010: 11).

Esti panorama inflúi a los individuos $-\mathrm{y}$ muncho- na toma de decisiones: yá nun s'apuesta, por exemplu, por comprar una casa pa siempre, pa evitar nun poder movese d'ella; yá nun s'espera tanto pol amor de tola vida, porque na rede alcuéntrense milenta. De fechu, preocupa prevenir que les coses seyan tan «sólides», tan fixes, que nun puean camudar nel futuru (Barranco Barcelona 2017). Presta más satisfacer les necesidaes al momentu, el bienestar caducu ya inmediatu, una actividá febril que tresforma la vida nun perpetuum mobile (Simmel 2010: 12), pero que nun sacia a los individuos dafechu.

Nesta «xaula de la modernidá» (Beck 1997/1998: 144), la inseguridá xenera una sensación de transitoriedá, de precariedá esistencial y d'inestabilidá constantes, un sentimientu de vacíu qu'intenta suplise col gastu,

\footnotetext{
3 Anguaño apaez un escesu de nuevos nomes pa nomar esos camudamientos: la «sociedá'1 riesgu», la «sociedá la información», «posindustrialismu», la «era comunicativa», la «segunda Edá Media», amás d'aquellos qu'amiesten un calificativu o prefixu a lo qu'había antes, como «modernidá tardía», «alta modernidá», «metamodernidá», «hipermodernidá», «supermodernidá» y, por supuestu, «posmodernidá» (Lyon 2000: 76).
} 
al percibise'l mundu como una superposición de productos pal consumu inmediatu (Bauman 2000/2004, 174). En pallabres del filósofu y escritor Javier Barraycoa, ye como si esta sociedá del mundu globalizáu tuviere que recurrir al consumu continuáu p'alcontrar nelli una identidá, falsa, pero que ye identidá a la fin (Barraycoa 2017). Sofita la postura'l sociólogu David Lyon, al comentar que la cultura del consumidor conviértese en centru moral y cognitivu de la vida, como forma de que les persones s'integren na sociedá (Lyon 2000: 155), polo xeneral por razones de seguridá emocional y sicolóxica (Bauman \& Donskis 2013/2015: 13).

\section{LA VIDA «ONE TO ONE»}

Nesta «comunidá global de consumidores» (Bauman \& Donskis 2013/2015: 74) too acaba por mercantilizase: cualesquier realidá que nun suponga una rentabilidá o que nun tea suxeta al consumu nun tien cabida:

La globalización pon en marcha un procesu d'interconexón a nivel mundial, que conecta tolo que val de calter instrumental -empreses, instituciones, individuos-, al tiempu que desconecta tolo que, pa esa razón, nun val (Martín-Barbero 2002: 57).

Por esi motivu, los especialistes en marketing camudaron l'enfoque de les estratexes de consumu a lo cabero del sieglu XX, pasando d'una producción masiva y estandarizada basada nel productu o «marketing de mases», a una aproximación centrada nel cliente y nes sos necesidaes, nomada «marketing rellacional» (Sánchez Arrieta 2007: 47). La diferencia ente ún y otru ye que'l «marketing de mases» trata al cliente «como un enemigu al que conquistar» (DD.AA. 2005: 103), demientres que'l marketing individualizáu apóyase nelli y míralu más como un collaborador.

Agora, colos mercaos colapsaos de competidores y cola facilidá d'accesu a los productos, ye necesario ser más qu'orixinales: estudiar lo que necesita l'homo consumens (Bauman 2003/2005: 72) y alcontrar nuevos «nichos de mercáu» (Lyon 2000: 110), ye dicir, espacios pequeños qu'inda nun tean atendíos, pa poder cubrilos col diseñu de productos personalizaos (Sánchez Arrieta 2007: 49). Ye, poro, imprescindible fixar un target menos ambiciosu y más seguru, que permita a les empreses sobrevivir nesta dómina cambiante.

D'esta miente surden, d'un llau, dellos modelos complementarios de «marketing rellacional», como'l One to One (Peppers y Rogers 1993), basáu nel estudiu personal del cliente o como'l modelu mass customization, centráu na diferenciación del productu (Pitta 1998). Y, d'otru llau, orixínense estratexes que revolucionen el potencial de les persones emprendedores, 
como'l modelu de creación de pequeñes empreses Business Model You, que combina'l citáu One to One cola creación d'una rede d'aliances interdependientes ente les persones d'esti colectivu. (Sánchez Arrieta 2007: 80). Inda más lloñe, popularícense metodoloxíes de trabayu que busquen, amás de camudar la empresa, revolucionar el sector nel que compiten, al da-y más importancia al valor del cliente: ye'l casu de la estratexa lean, desendolcada pola compañía xaponesa de fabricación d'automóviles Toyota dende los años 80 del sieglu XX y estendida anguaño al restu de sectores.

La metodoloxía lean constrúi una filosofía de pensamientu dinámica y distinta a la estructura conceptual tradicional: pasa de pensar nos trabayadores como máquines que siguen el mandatu de lo que «tien que facese», a confiar na so creatividá pa poder identificar xuntos los problemes inmediatos y decidir l'orde de prioridá pa resolvelos. Dende esti puntu de vista, sofítase un sistema de deprendizaxe onde se desendolquen desafíos pa superar los retos más difíciles y onde s'analicen les oportunidaes pa satisfacer les necesidaes cambiantes (Ballé, Jones, Chaize \& Orest 2017/2018: 61). Les persones que lo posibiliten caracterícense por dir desarrollando un compromisu individual al rodiu d'esti proyectu, con una iniciativa y una creatividá que son escuchaes y valoraes dientro d'una organización dinámica ya interconectada, amestando otres capacidaes d'anovar. Dende esta perspectiva, el cliente yá nun ye un consumidor «qu'estruyar», sinón una persona «a la qu'ayudar» (2017/2018: 125).

De los modelos y estratexes de mercantilización que se tán describiendo nun se decaten solo les empreses. La investigación y la educación tán tamién mercantilizándose (Zufiaurre 2007: 12): el pasu d'una economía basada na industria a una afitada nel conocimientu (Fernández 2008: 14) esixó una modificación de los planes d'estudios y una redefinición del papel de les universidaes como hologrames sociales. El «mercáu de la educación» (Bauman 2012: 107) diseña yá itinerarios flexibles y adaptables al gustu del estudiantáu, de mou que cada persona crea un camín a la carta (Lyotard 1991: 40) y potencia'l so valir nun sistema y ecosistema de deprendizax ecolóxicu. La concepción de los estudios universitarios como negocios fai del estudiantáu un potencial cliente $^{4}$, utilizando la xíriga empresarial, llegando a ser calificada la guía docente como'l contratu que tien esti colectivu col profesoráu. Diz nesta dirección el maestru d'Educación Primaria y Llicenciáu'n Filoloxía Inglesa, César Bona, nomáu ente los 50 meyores docentes del mundu nel añu 2015, que de fechu la deshumanización

\footnotetext{
${ }^{4}$ Exemplu d'ello ye la siguiente afirmación testual, cuasi visionaria, que s'atopa yá nun volume de pedagoxía de la UNED nel añu 1994: «Consideramos la práctica de la enseñanza como una profesión abierta y de proceso y al profesor a un profesional, sin obviar los problemas, y por tanto los campos a ir conquistando, que aparecen en su grado de autonomía, la complejidad del mundo del cliente y el control intra e interprofesional» (Gairín Sallán 1994: 101).
} 
del pensamientu educativu ta algamando unes llendes inimaxinables (Bona 2016/2017: 12). La universidá d'agora flota ente les demandes de les novedaes del mercáu, de la política y de la opinión popular, una situación que'l profesor de Filosofía de la Educación Jorge Larrosa noma «capitalismu cognitivu» (Larrosa 2019: 12).

Y, d'otra banda, adáptense tamién a los modelos rellacionales los mass media y les principales redes sociales, como Facebook o Instagram, ufiertando la posibilidá de silenciar lo que nun interesa al dueñu del perfil y poniéndo-y en primera llinia lo que l'usuariu quier ver. Estes plataformes entruguen al usuariu si-y presten determinaes publicidaes o non, pa encamenta-y otres más afayadices. Y tampoco nun se puen escaecer les cookies, qu'apaecen cuando l'usuariu abre una páxina web, pa rexistrar los itinerarios de consulta y de compra que-y llamen más l'atención.

Ye tan altu'l nivel de consumu de la «modernidá líquida», que cualesquier individuu pue especular coles posibilidades de convertise nuna persona influyente creando una imaxe pública que respuenda a los ideales publicitarios del momentu, mercantilizando la propia privacidá y promoviendo'l cultu a la novedá, a la diversión y al bienestar ente la xente mozo (Bauman 2012/2013: 43), per aciu de la cercanía y de la emocionalidá (Calvo 2016: 66). Trátase de la inmersión nel mundu del consumu más directa y estensiva qu'esistió hasta güei (Bauman 2012/2013: 64): agora calcúlase a qué hora ye meyor colocar una semeya nel perfil d'Instagram pa consiguir más impactu; y, en dellos casos, sácase rindimientu económicu d'estes práctiques, col patrociniu de marques, ensin requerimientu de conocimientos previos del mercáu. Según un estudiu de 2018 sobre'l marketing d'Influencers n'España, un 67,8\% d'esti colectivu recibe yá ingresos derivaos de diches xeres (Brandmanic 2018: 12). Estes acciones nun son otra cosa que práctiques en rede onde se conecten y se combinen estayes de conocimientu qu'antes crecíen separtaes, como la tecnoloxía, la moda, la fotografía, la publicidá, la psicoloxía o la socioloxía. Funciónase asina como sociedá de consumidores y productores rizomáticos 3.0, xenerando, anota Bauman, un círculu d'ufierta y demanda pa consigo mesmos (Bauman 2013/2015: 41). Esti tipu de comportamientos fai posible que se dean en paralelo casos tan contradictorios como pensar nunu mesmu y vivir namái que pa los otros (Beck 1999/2006: 16).

\section{DEL SILENCIU AL ESPORPOLLE DE LES IDENTIDAES}

El globalismu del que surden les práctiques indicaes de producción y consumismu, lloñe de crear namái un efectu uniforme y homoxeneizador nel mundu, como podría pensase a priori, consigue evidenciar más la diferencia y 
la heteroxeneidá (Lyon 2000: 98). D’un llau, propón un procesu d'esclusión/inclusión que convierte la cultura nun espaciu estratéxicu d'emerxencia de les tensiones (Martín-Barbero 2002: 45). D'otra miente, a una sociedá cada vuelta más desterritorializada y nómada interésa-y el contactu con otres realidaes culturales y étniques (Barraycoa 2017), qu'abren l'abanicu de la esperimentación: nueves miraes, histories qu'ufierten un mundu distintu dientro del propiu mundu. Agora ye mayor la conciencia de los contrastes, al interactuar con ellos. Por eso, estos acaben buscándose, yá seya p'autoafirmar lo que se ye n'oposición al restu -ello quier dicir, por «impulsu de lo propio en rellación colo ayeno» (Barreiro Maceiras 2014: 220)-, o pa configurar una identidá a la carta y escaecer la propia (Bauman 1999b: 109). Porque, nesti momentu, les identidaes, entendíes como «procesos sociales en construcción» (Vergara 2006: 97) vense tamién atrapaes nos procesos de mercantilización (Rodríguez Pérez 2004: 256) y tórnense «identidaes liquides» (Roman Etxebarrieta \& Pérez-Izaguirre 2018: 317), identidaes «reconstruyíes a partir del movimientu social y del intercambiu ente persones que s'inflúin ente sí, de manera directa o indirecta» (Calvo 2016: 89).

Afondando más na cuestión, el significáu de cultura altérase anguaño y pasa a ser sinónimu d'identidá, un indicador y diferenciador de la mesma (Benhabib 2002/2006: 22). Créase una nueva conciencia de la diversidá y algamen protagonismu los discursos que traten les identidaes sexuales, étniques, llingüístiques o de cualesquier otru calter, silenciaos ${ }^{5}$ nes dómines anteriores. Los grupos que surden al rodiu d'estos «indicadores identitarios» esixen nesta dómina un reconocimientu llegal y una distribución de los recursos del Estáu y de los sos organismos pa preservar y protexer les sos especificidaes culturales. Esto asocede, apunta'l doctor en Comunicación Enrique Vergara (2006: 97), al constituir la identidá una de les principales fontes de seguridá personal, de movilización colectiva y de significación social: «la nuesa forma de posicionanos nel mundu» (Calvo 2016: 91). Desendólcase un procesu cognitivu pel que se creen na mente aliances y dicotomíes ente'l Nós y los Otros (Roman Etxebarrieta \& Pérez-Izaguirre 2018: 318). Y ye que, como comentaben yá los filósofos Deleuze \& Guattari al falar del rizoma, «nun hai una sola identidá, sinón identidaes móviles y diverses» (1971, apud López Gil \& Angulo Rasco 2015: 12) que se definen por dalgunes característiques, dellos símbolos o práctiques sociales en contraste.

$\mathrm{Na}$ mesma llinia, agora redefinese tamién l'alteridá ya interesa promulgar el multiculturalismu, entendíu como'l derechu del ser humanu a

\footnotetext{
${ }^{5}$ El silenciu apaez venceyáu a cuestiones identitaries en munchos contestos sociales opresivos (Llamedo 2019).
} 
escoyer la identidá propia (Bauman 2001: 111). D'esta miente, dase cabida a un mercáu de les voces silenciaes poles dómines anteriores y a un puxu polos discursos amenorgaos, cuando'l reconocimientu pasa a ser consideráu dientro de les operaciones fundamentales pa la constitución de les identidaes (Giménez 2009). A esti respectu, diz el calderalgu de Didáctica Benjamín Zufiaurre que nel marcu d'una sociedá más global, dase la paradoxa de que les sociedaes reivindiquen mecanismos de discriminación positiva pa poder ser más democrátiques (Zufiaurre 2007: 23). Sofita esta idea Martín-Barbero, al dicir que «lo que galvaniza güei a les identidaes como motor de llucha ye inseparable de la demanda de reconocimientu y de sentíu» (Martin-Barbero 2002: 57). Y conclúi Bauman al respectu, que xusto en nenguna otra dómina se sintió d'un mou tan fondu la necesidá d'escoyer baxo l'amenaza constante de «quedar na cuneta»y «ser excluyíos del xuegu» (Bauman 2012/2013: 29).

\section{LA NORMALIZACIÓN DEL ASTURIANU Y DEL GALLEGO-ASTURIANU}

\subsection{Dilemes actuales}

Magar que se dea una creciente necesidá d'escoyer, ello nun quier dicir que la escoyeta seya una actividá cenciella. Amuesa esta circunstancia'l casu de la normalización de les llingües gallegoasturiana y asturiana. Falar na modernidá actual de dos llingües romániques inda minorizaes y en situación de diglosia sigue siendo complexo, anque esistan colectividaes que dean güei puxu a les voces minorizaes o a les identidaes arrequexaes nes dómines anteriores, ya inclusive contando con que la conciencia identitaria n'Asturies ye mui fuerte y apaez descrita como una de les más sólides del estáu español (González-Quevedo 2019: 72). Plantégasenos equí un primer dilema: ¿cómo ye posible?

El primer motivu, como indica'l filósofu, antropólogu y llingüista Roberto González-Quevedo, ye políticu: esa fuercia identitaria tradúxose en pocos casos en resultaos electorales que permitieren el sofitu d'una representación parlamentaria. Ensin esa posibilidá, nun foi posible llegar a una situación de co-oficialidá, como sí asocedió por embargu nel casu d'otres llingües minorizaes del estáu español.

El segundu motivu ye la dificultá qu'esiste güei por aceptar cualesquier compromisu, mayor que nes décades anteriores. La propuesta de la co-oficialidá p'algamar una «situación de normalidá sociollingüística» consiste nun discursu de calter «sólidu» ufiertáu a una sociedá, como se vien diciendo, cada vuelta más «líquida» y más insegura, d'algo que nun ye esclusivo de la nuesa comunidá llingüística, sinón del conxuntu de la sociedá mundial, empero 
qu'asocede con más intensidá nel momentu de mayor percorríu normalizador de les nueses llingües. Esta «globalidá irreversible» (Beck 1997/1998: 146) provoca que l'esfuerciu por comprender la cuestión, asina como la implicación nes xeres de manifestación d'una llealtá llingüística, nun apaezan siempre como prioritaries. Invertir tiempu y esfuerciu nos procesos complexos y duraderos nun paga del too la pena pa la sensibilidá moderna (Simmel 2010: 8), porque l'homo aeconomicus y l'homo consumens manifiéstense como individuos ensin atadures sociales (Bauman 2003/2005: 96), qu'alcuentren la so satisfacción no provisorio cuando les metes nun s'algamen rápido. Dicen, nesti sentíu, Bauman y Donskis que nun sorprende la desllealtá nun mundu de venceyos intermitentes (Bauman \& Donskis 2013/2015: 370).

Siendo estes les circunstancies, la pluralización y la fragmentación de les sociedaes consumidores actuales ponen a prueba les concepciones actuales de verdá y minen los umbrales de tolerancia, que pasen a ser muncho más superficiales y de curtiu alcance, hasta llegar a disputar inclusive la verdá de la ciencia (Lyon 2000: 119). Alviértese equí un tercer motivu deriváu d'esti fechu: la proliferación de discursos carentes de rigor científicu sobre la llexitimidá de les llingües gallegoasturiana y asturiana y l'aprovechamientu d'estes circunstancies pa la creación de campañes polítiques escontra la so cooficialidá. Ye exemplu d'ello el discursu actual del portavoz de Vox, Ignacio Blanco, qu'acusó, nel mes de marzu de 2019, a l'Academia de la Llingua Asturiana de persiguir col so trabayu oxetivos de calter nacionalista ayenos a la co-oficialidá de les llingües citaes (Rojo 2019a) y, nel mes d'ochobre del mesmu añu, estendió tamién l'acusación a los partíos políticos d'izquierda sensibilizaos cola cuestión llingüística (Rojo 2019b).

Un segundu dilema que se presenta equí ye'l calter del plan estratéxicu pal espardimientu y pa la reivindicación de la co-oficialidá de les dos llingües minorizaes mentaes. Valga d'exemplu'l casu del asturianu, por contar yá con una codificación del corpus más desarrollada. Repasando los cuatro pegollos qu'afiten una llingua y qu'acaben coles condiciones de diglosia trazaes por Ferguson nel 1954 (Díaz Trancho 2014: 13) -esto ye: que tea normativizada pa garantizar la normalización del corpus y el so conocimientu, qu'esista una tradición lliteraria nesa llingua, que seya funcional en tolos contestos y que s'estandarice y se normalice socialmente-, anguaño llúchase por afitar el cuartu y últimu, porque los otros tres son yá una realidá. Apaez nesti cuartu nivel el problema que sigue: les estratexes que se necesiten pa consiguir la normalización social d'una llingua nun puen ser les mesmes que s'utilizaron nos otros casos, porque la naturaleza de los cuatro pegollos ye diferente. Más en concretu, pa la normativización y pa la «cultivación léxica» y «estilística» (D'Andrés 2018: 29) requeríase un trabayu fundamentalmente d'estudiu, d'investigación, de gabinete y de toma de decisiones, por parte de les persones 
cualificaes, como asina se fixo. Pa la xeneración d'una tradición lliteraria, too dependía d'una voluntá creativa individual del usuariu de la llingua que tuviera esa capacidá, esa sensibilidá y esi amor pola escritura, daqué más arbitrario, pero nesti casu real; y, pa la so promoción, foi otra vuelta necesaria la filoloxía y la historia. Empero pa la normalización llinguística, ye necesaria la intervención de más campos de la ciencia -sociollingüística, socioloxía del llinguaxe, antropoloxía, política, comunicación, marketing-, dende que ye imprescindible garantizar la llealtá llínguiística de la comunidá de falantes (Galán \& González 2014: 219; Guardado Díez 2008: 149). Esto ye, tien que se contar col alcuerdu de la sociedá civil y de les sos fuercies polítiques mayoritaries (Barreiro Maceiras 2017: 29), pa que se tomen midíes llegales con influxu na conciencia social ciudadana que garanticen tamién la tresmisión xeneracional. Esti requerimientu amplía forzosamente'l círculu de persones implicaes nel procesu de normalización llingüística y multiplica les variables d'inseguridá. Algamar esti últimu pegollu ye, polo tanto, una xera muncho menos previsible dende'l puntu de vista científicu, menos sólida que les anteriores y más rizomática, porque hai que tener en cuenta les variables que se dean na sociedá, cambeos que nel mundu d'agora son difícilmente estimables.

Ye cierto que la plena normalización y el refuerzu de la llealtá llingüística necesiten tamién d'una planificación del corpus y del estatus y d'una implicación institucional (Guardado Diez 2008: 150) qu'igüe un aparatu llexislativu en consecuencia, empero nun se van poder decidir namái al rodiu d'una mesa d'espertos nin solo con una perspectiva filolóxica: cuando se propón l'usu y la co-oficialización de cualesquier de les dos llingües a partir de la esposición de razonamientos «sólidos» y xerárquicamente organizaos, ponse nel centru el productu, la llingua, como se facía nel marketing de mases tradicional. De mou y manera que s'atopa como receptora a una sociedá líquida y rizomática que nun lo comprende, que s'apavoria coles realidaes incombustibles, colo duradero, colo perenne: apez una sociedá que transita pel mundu nuna incesante busca de la propia identidá, que nun toma decisiones de nengún tipu, nin nel planu más personal y priváu de la so vida. Una sociedá que s'alloña de los posicionamientos qu'impliquen compromisu y que s'avera a discursos diferentes pol fechu de probase a sí mesma, pero d'un mou polo xeneral superficial. D'esta miente, el procesu de normalización tórnase mui «lentu» (DD.AA. 2018: 174) y «poco eficaz» (González-Quevedo 2019: 71), porque proponer un cambéu «sólidu» significa tener que dir a contracorriente colos tiempos. Falando códigos sociales estremaos orixínense unes distancies de pensamientu complicaes de salvar.

\subsection{Estratexes posibles}

¿Cuál ye, entós, la solución pa salvar les distancies cola masa crítico? 
Anque la fórmula máxica nun esiste, cuídase útil considerar, dientro de los saberes de la socioloxía, «el balance social global» que se desprende de les iniciatives de normalización (González-Quevedo 2019: 60), non solo de puertes p'adientro - como se vien faciendo col analís de les movilizaciones sociales poles llingües na historia d'Asturies o coles estadístiques sociollingüístiques rexonales-, sinón tamién atendiendo a les preocupaciones y a les prioridaes del falante como individuu social d'anguaño. Analizar y comprender el mundu d'agora y la sociedá que lu habita pue resultar difícil pola so complexidá pero tórnase una ventaxa porque, refieren Bauman y Donskis, «la llealtá ye la estratexa de descubrir el mundu xuntos» (Bauman y Donskis 2013/2015: 264): la llealtá llingüística tien de trabayase en conxuntu. Aceptar el caos y almitir la confusión, como propón Lyon (2000: 149) puen ser formes de tresformar el comportamientu de la modernidá.

Dende esti puntu de vista, la estratexa más granible acaba por ser falar col llinguaxe que la «sociedá líquida» entiende, el que manexa, el que ta avezada a oyir y nel que participa: normalizar les llingües gallaegoasturiana y asturiana dafechu nun mundu onde'l consumu ye prioritariu pasa per saber deprender de los plantegamientos del «marketing rellacional», no tocante al mou de comunicase colos falantes y de da-yos voz, como faen les empreses colos clientes dende va treinta años, como lleven faciendo les universidaes col estudiantáu y los medios de comunicación colos usuarios pelo menos nos últimos quince.

Más en concretu, tocaría reflexonar sobre si los modelos de normalización social y de llamamientu a la co-oficialidá que se tán realizando casen más coles estratexes de «marketing de mases» de la vieya «modernidá sólida», onde la co-oficialidá trataríase como un productu colectivu preparáu pa calar por igual en tola xente o si, por embargu, esi procesu ta centrándose nel falante invidual de la llingua, como fadríen una postura más «rellacional one-to-one»o un pensamientu lean: ¿tamos concentrándonos no qu'al falantey preocupa, pa consiguir empatizar y que sienta la so llingua minorizada afayadiza? ¿Qué torgues alcuentra? ¿En qué estayes de la so realidá diaria podría dise incorporando pa potenciar el so usu?

Una prueba de la efectividá d'esti tipu d'aproximaciones al falante alcuéntrase nel sector de los medios de comunicación asturianos, quiciás por tratase d'un ámbitu más cercanu al marketing y, polo tanto, nel que se conoz esti llinguaxe y onde hai una consciencia más fuerte de la necesidá d'esti saltu hacia una fase rellacional. Valgan d'exemplos d'esta denuncia activa y del analís nos sectores audiovisual y de prensa escrita de profesionales con una bayurosa esperiencia, como son los periodistes Cristóbal Ruitiña ya Inaciu Galán y González. 
D'una banda, Cristóbal Ruitiña lleva dellos años denunciando los escasos resultaos del impactu de la Radiotelevisión del Principáu d'Asturies na normalización social del asturianu y del gallegoasturianu. Ruitiña da cuenta de la falta de criterios de programación horaria ${ }^{6}$ y de conteníos nestes llingües inda en determinaos formatos, lo que provoca un «ruin efectu normalizador» de la RTPA (Ruitiña 2012: 236). Confírmalo'l periodista Damián Barreiro al concluir que, nos primeros diez años de vida, la televisión pública asturiana valió más bien pa reforzar la imaxe folclorizada y antropolóxica de la identidá asturiana, manteniendo l'estatus de llingües minorizaes de los idiomes (Barreiro 2017: 29). La empresa pública tien una potencialidá y un alcance muncho más aprovechable, sobre too dende que ta xeneralizada la sintonización, a raíz de les tresmisiones de la «Fórmula Uno» de los años 2011 y 2012. Por esi motivu Ruitiña espón la necesidá d'afianzar la producción industrial de les llingües minorizaes a traviés los medios de comunicación como RTPA (Ruitiña 2013: 130), considerando que se puen diseñar meyor los «nichos de mercáu» que seyan necesarios con esti oxetivu.

Ruitiña acercáse asina a los conceptos rellacionales de la xíriga empresarial, al referise al camudamientu de la percepción que se tien de la utilidá les llingües y al atender tamién a la necesidá d'acomodase al imaxinariu mercantil de la sociedá, tanto pol bien de los impulsores del sector audiovisual (Ruitiña 2013: 129), como pol bien del futuru de la llingua:

Una llingua sobrevive mientres ye útil. Históricamente, una llingua yera útil cuando sirvía pa qu'un emisor y un receptor se comunicaren. Más modernamente, una llingua tamién ye útil cuando una determinada sociedá llogra industrializar el so caltenimientu y reproducción. (Ruitiña 2013: 129)

Y desplica cómo, inda cuando nun s'inclúin munchos conteníos, por exemplu, en llingua asturiana nos horarios de máxima audiencia estos, polo xeneral, ameyoren la media de datos de la cadena pública asturiana (Ruitiña 2012).

Amás, completando esti discursu, RTPA tampoco nun ufre «una llingua asturiana modélica y dignificada al traviés programes de prestixu» (DD.AA. 2018: 147), lo que ye un derechu que figura na «Llei 1/98, de 23 de marzu, d'Usu y Promoción del Bable/Asturianu»; como tamién lo ye que l'asturianu [y el gallegoasturianu] tenga «una presencia amañosa» y heteroxénea neses emisiones (Barreiro Maceiras 2014: 232), pa dar respuesta a la demanda que faen al respectu seis de cada diez asturianos (Llera Ramo 2018: 18) y, más en concretu, a la de cuasi'l 80\% de los mozos y moces (Hevia Artime 2018: 16).

${ }^{6}$ Confirma la doctora n'Investigación en Medios de Comunicación, Azahara Cañedo, que nel períodu 2006-2014 la mayor parte de los programes n'asturianu (53\%) ubicóse na estaya de la mañana (Cañedo 2018: 432). 
Al contrario, son munches les ocasiones nes que s'amiesten rexistros y códigos, una práctica qu'asocede tamién n'otros escenarios d'históricu conflictu llingüísticu, como l'occitanu, onde la publicidá de dellos productos que se fai en francitanu (Alén Garabato 2019: 193) nun ye otra cosa que l'aceptación y el refuerzu d'una situación diglósica de la que paez que nun se quier salir.

La «dexadez de funciones» actual de RTPA (Barreiro Maceiras 2017: 32) provoca un déficit grave «de responsabilidá de los medios de comunicación públicos del Principáu (DD.AA. 2018: 147), «una situación alarmante y allegal» (XDLA 2013: 23), si se tien en cuenta la importancia que puen algamar estos medios de comunicación pa «garantizar una penetración social mayor» (Ruitiña 2012: 229), contribuyir a la normalización social d'una llingua minorizada (XDLA 2013: 3) a acabar col estrañamientu y crear «una opinión pública compartida» (Barreiro Maceiras 2014: 224), sobre too nesta dómina de conexones globales. Diz a esti respectu Vergara, que «ye nel contestu de la complexidá qu'alquier la globalización y les sos tensiones culturales onde los mass media consiguen un protagonismu indiscutible nes construcciones de nueves visiones del mundu» (Vergara 2006: 96). La espansión de los mass media na sociedá de consumu contribúi a popularizar la llingua dominante d'un llugar «d'una forma esponencial» (Guardado Díez 2008: 78), al facese propaganda de les ideoloxíes mayoritaries, que suelen tar venceyaes a la tendencia económica que mantién esos medios (Calvo 2016: 70). Por eso, cuídase necesario que'l restu de llingües que nun seyan mayoritaries tean mui presentes pa nun quedar entá más arrequexaes pa que los sos falantes tean involucraos nel procesu.

D'otra banda, otru de los profesionales más concentraos nel sector de la comunicación, Inaciu Galán y González, demandaba yá nes Xornaes d'Estudiu de 2011 la falta de recursos periodísticos en llingua asturiana (Galán y González 2011), asina como l'escasu sofitu institucional nesi sen. Sicasí, nel 2014, el periodista reconocía'l surdimientu de les nueves tecnoloxíes como una oportunidá pa dar a la llingua asturiana «espacios nuevos de normalidá» (Galán y González 2014: 208), entrugando directamente a los usuarios poles sos preferencies na rede como internautes asturfalantes. D'esta miente, Galán y González decidió aprovechar eses ferramientes pel mediu de la creación d'una pequeña empresa productora pa xenerar conteníu adaptáu a les demandes sociales y a les necesidaes del usuariu: la nomada «CuatroGotes Producciones SL» (2018). Esti intentu d'acercase al individuu falante como cliente y entender lo que-y interesa o preocupa percíbese yá na páxina d'aniciu de la web con una llamada n'asturianu espresada de tu a tu, «Hola! Yá tas en CuatroGotes. Agora cuéntanos» (CuatroGotes Producciones SL 2018), que ye, ensin dubia, una 
estratexa de marketing rellacional. Los resultaos d'esta empresa, qu'amás de producción d'audiovisual proyecta otros servicios personalizaos pal falante, nun tán tardando n'apaecer: collaboraciones col Principáu d'Asturies, colos Ayuntamientos de Xixón y d'Avilés o cola RTPA, ensin escaecer el premiu Gava 2019 pola creación de la plataforma dixital PlayPresta, na que'l falante d'asturianu pue escoyer conteníos d'entretenimientu de distintu tipu. La so iniciativa, ensin entrar nel beneficiu priváu que puea algamar, ye un exemplu de cómo l'averamientu al falante asturianu con un llinguaxe de calter «rellacional», más cercanu, gusta, porque crea una «imaxe de marca» (Méndez \& De Carbexe 2000: 29) como seña de diferenciación y de reconocimientu de la so llingua na que los individuos s'alcuentren y s'afayen. Por esi motivu, emplegar estes aproximaciones dientro de la esfera del poder públicu ya institucional, asina como de l'acción colectiva non institucional que vela pola normalización social de la llingua asturiana, podría acelarar esti procesu per aciu de la «promoción directa» (Guardado Díez 2008: 134), fomentar el so usu ya incrementar la curiosidá de la comunidá llinguística, al reforzar una «conciencia metapragmática común», pola que se reconozan como usuarios d'una mesma llingua (González Rodríguez \& Saavedra Fernández-Combarro 2006: 67).

Estos itinerarios que dibuxen, ente otros profesionales, Ruitiña y Galán, paecen axustase más a la realidá esistente nel camín hacia la normalización de les llingües asturiana y gallegoasturiana dientro del panorama social actual, como reacciones empobinaes a salvar les distancies qu'esisten cola masa crítica de falantes y potenciales falantes. La cuestión que se plantega ye si sedría preferible tardar más tiempu en poner remediu a eses distancies aprendiendo por ensayu-error, como nel casu de la RTPA que denuncia Ruitiña, o si resultaría más granible aprovechar dende yá les ferramientes a disposición que conceden estos tiempos modernos pa planificar y axilizar la normalización social con una perspectiva más rellacional, como fai la empresa de Galán colos medios audiovisuales.

Sicasí, el retu que se propón nun ye simple: el desendolcamientu xeneralizáu d'un modelu de pensamientu de calter rellacional requier siempre un sofitu institucional y políticu pa que se garantice una homoxeneidá de perspectives y una concentración de toles fuercies actantes que trabayen na mesma llinia. Nun se pue escaecer que la normalización social de cualesquier llingua supón un procesu institucional (González Riaño 2002), que ye responsabilidá del poder políticu que gobierna'l territoriu onde ta allugada cada comunidá llingüística y, por esi motivu, almítese como necesaria la toma de conciencia de dichu gobiernu pa consiguir trabayar en conxuntu cola sociedá y col restu d'organismos normalizadores. La situación actual paez precisamente afayadiza pa dar el pasu llexislativu pertinente y poder iguar una 
estratexa de normalización de calter rellacional, no que se refier a la consideración del falante como suxetu de pensamientu y d'opinión activu. Les circunstancies que lo corroboren son munches: güei hai un interés mundial pol plurillingüismu, l'averamientu a la tecnoloxía ta abriendo horizontes (Galán y González 2014) y les llingua asturiana y gallegoasturiana ganen aceptación y usu, como amuesen a nivel social social les últimes encuestes sociollingüístiques d'Asturies (Hevia Artime 2017; Llera Ramo 2017; Informe 2006) o, nel ámbitu políticu, la creación de la Conseyería de Cultura, Política Llingüística y Turismu del Principáu d'Asturies en 2019. Nun hai que escaecer, amás y como dixeron nel entamu del sieglu XXI Méndez \& De Carbexe (2000: 29) pal casu del ámbitu territorial Navia-Eo, que los exes de rellaciones que se creen ente'l patrimoniu llingüísticu, la sociedá que compón estes comunidaes de falantes y les economíes llocales configuren un contestu afayadizu pa que estes llingües alquieran un pesu rellacional y emocional perimportantes.

\section{A MOU DE CONCLUSIONES}

Nestos «tiempos líquidos» (Bauman 2007) de conexones rizomátiques, pluralidaes culturales ya inseguridaes sociales, la modernidá avérase a lo que resulta funcional y entrúgase pola utilidá de tolo que-y repercute. A esi respective, esta colectividá fuxe de zarrar un futuru fixu y prefier movese pela llinia de lo caduco.

Les comunidaes llingüístiques favoratibles a les llingües asturiana y gallego-asturiana, mentanto, lluchen pola so co-oficialidá, p'algamar un billingüismu equilibráu perenne y desaniciar la diglosia, dempués de completar un llargu camín d'analís internu y esternu, de normativización y d'asoleyamientu y difusión d'una granible tradición lliteraria.

Cuando lo híbrido y lo líquido pasen a sustituir la «rixidez cultural» y la «llingüística pretérita» (Roman Etxebarrieta \& Pérez-Izaguirre 2018: 318), alcuéntrese nel camín estes dos realidaes paraleles: un «sólidu» llabor de normalización social d'una ferramienta llingüística y una sociedá más «líquida» que nunca. Entren asina en conflictu el compromisu cola tendencia xeneral al individualismu.

Cuídase, por esti motivu, imprescindible, alcontrar un entendimientu ente dambos llinguaxes. Ufiértase, en consecuencia, como estratexa dinámica de diálogu l'aprovechamientu de patrones d'atención al falante y d'aproximación rellacional basaos nos modelos que nacieron col marketing individualizáu One to one y coles estratexes lean de trabayu de les multinacionales, patrones que yá se tán probando n'otres esferes de la vida y que tán dando frutos. Apúrrense, a mou d'ilustración, exemplos concretos 
dientro del panorama de la normalización social del asturianu nos que l'usu más o menos consciente d'estratexes rellacionales comencipia a algamar rápido resultaos granibles, demostrando que llevar esta teoría a la práctica ye posible.

Con esta descripción teórica, lloñe de zarrar la cuestión, preténdese abrir una ventana nel estudiu sociollingüísticu y sociolóxicu de les llingües minorizaes asturiana y gallegoasturiana, enanchando la mirada hacia l'aprovechamientu tresversal de les distintes estayes del conocimientu humanu pa contribuyir al so desendolcamientu llingüísticu y a completar la so normalización. Sedría conveniente realizar agora estudios qu'apurran datos empíricos sobre les estratexes rellacionales de normalización que son yá una realidá, teniendo mui presente'l marcu conceptual espuestu, relativu al rizoma y a les modernidaes, que sedrá ampliáu en rellación con otres cuestiones nuna futura tesis doctoral.

\section{BibLIOGRAFÍA}

\subsection{Fontes impreses}

Alén Garabato (2019): Carmen: «El Occitano en el siglo XXI: ¿la utopía de la normalización?», en Javier Giralt Latorre y Francho Nagore Lain (eds.), La normalización social de las lenguas minoritarias. Experiencias y procedimientos para la salvaguarda de un patrimonio inmaterial. Zaragoza, Universidad de Zaragoza: 181-209.

ANDRÉS DÍAZ, Ramón d' (2018): «Lenguas minoritarias y estandarización», en Javier Giralt Latorre y Francho Nagore Lain (eds.), Lenguas minoritarias en Europa y estandarización. Zaragoza, Universidad de Zaragoza: 19-45.

BALLÉ, Michael, Daniel Jones, Jacques Chaize y Orest Fiume (2017/2018): Estrategia Lean. Utilizar lean para crear ventaja competitiva, generar innovación y facilitar el crecimiento sostenible. Editorial Profit. [ $1^{\mathrm{a}}$ ed. en castellán].

BARREIRO MACEIRAS, Damián (2014): «La nación televisada: llingua y identidá nos programes n'asturianu de la Televisión del Principáu d'Asturies», en Lletres Asturianes 111: 219-253.

- (2017): El papel de TPA na normalización de la llingua asturiana. (Trabayu de Fin de Máster). Barcelona, Universitat Oberta de Catalunya. [Consulta a 9/10/2019, recuperáu de http://hdl.handle.net/10609/66766]

Bauman, Zygmunt (1999a): En busca de la Política. México, FCE México.

- (1999b): La globalización. Consecuencias humanas. México, FCE México.

- (2000-2004): Modernidad líquida. Buenos Aires, FCE Argentina. [3 $3^{\mathrm{a}}$ reimp. de la $1^{\mathrm{a}}$ ed. en castellán].

- (2001): La sociedad individualizada. Madrid, Cátedra.

- (2003-2005): Amor Líquido. Acerca de la fragilidad de los vínculos humanos. Madrid, FCE España. [1 $1^{a}$ ed. en castellán].

- (2007): Tiempos líquidos. Vivir en una época de incertidumbre. Barcelona, Tusquets Editores.

- (2012): Esto no es un diario. Madrid, Espasa. [1 ${ }^{\mathrm{a}}$ ed. en castellán].

- (2012-2013): Sobre la educación en un mundo líquido. Conversaciones con Ricardo Mazzeo. Barcelona, Paidós. [ $1^{a}$ ed. en castellán].

BAUMAN, Zygmunt y Leonidas DONSKIS (2013-2015): Ceguera moral. La pérdida de 
sensibilidad en la modernidad líquida. Barcelona, Paidós. [1 $1^{\text {a }}$ ed. en castellán].

BECK, Ulrich (1997-1998): ¿Qué es la globalización? Falacias del globalismo. Respuestas a la globalización. Barcelona, Paidós. [De toles ediciones en castellán].

- (1999-2006): La sociedad del riesgo global. Madrid, Siglo XXI de España. [2a ed.].

BenHABIB, Seyla (2002-2006): Igualdad y diversidad en la era global. Buenos Aires, Katz Editores. [ $1^{\mathrm{a}}$ ed.].

Bona, César (2015): La nueva educación. Los retos y desafíos de un maestro de hoy. Barcelona, Penguin Random House.

Calvo González, Soraya (2016): Identidades (no solo) digitales. Aproximación al modelo de comunicación afectiva en redes sociales de la juventud asturiana. Implicaciones para la intervención socioeducativa. (Tesis doctoral). Uviéu, Universidá d'Uviéu. [Consulta a 30/10/2019, recuperáu de http://hdl.handle.net/10651/40604].

CAÑEDO, Azahara (2018): Televisión del Principado de Asturias: dinamización y diversidad en el sector audiovisual asturiano (2005-2015). (Tesis doctoral). Madrid, Universidad Carlos III. [Consulta a 9/10/2019, recuperáu de http://hdl.handle.net/10016/27512 ].

CORMIER, David (2008): «Rhizomatic education: Community as curriculum», n'InnovateJournal of Online Education, 4 (5). [Consulta a 02/12/2019, recuperáu de http://davecormier.com/edblog/2008/06/03/rhizomatic-education-community-as-curriculum].

DD.AA. (2005): «Estrategias de Marketing y Clientes», en Diario Expansión. Madrid, Daemon Quest.

DeleuZe, Gilles y Félix GuATTARI (1977): Rizoma (Introducción). Valencia, Pre-textos.

Díaz Trancho, Iris (2014): Duermi fíu del alma. La tresmisión xeneracional d'una llingua en peligru: el casu asturianu. (Trabayu de Fin de Máster). Uviéu, Universidá d'Uviéu. [Consulta a 7/10/2019, recuperáu de http://hdl.handle.net/10651/46529].

FERNÁNDEZ MARCH, Amparo (2008): «La innovación de la docencia como estrategia de cambio ante los desafíos del EEES», en María Esther Moral Pérez \& Raquel Rodríguez González (coords.), Experiencias docentes y TIC. Uviéu, ICE Universidá d' Uviéu: 9-42.

GAIRín SALLÁN, Joaquín (1994): «La profesionalización docente y su influencia en la vida institucional», en Ramón Pérez Pérez (coord.), Calidad de vida en los centros educativos. Xixón, Centru Asociáu de la UNED de Asturies-Xixón: 101.

GALÁN Y GONZÁLEZ, Inaciu (2011): «Periodismu audiovisual nuna llingua minorizada: delles reflexones sobre'l casu asturianu», en Lletres Asturianes 105: 121-133

- (2014): «L'asturianu y les nueves teunoloxíes: datos d'una encuesta d'usos llingüísticos», en Lletres Asturianes 110: 207-226.

García ARIAS, Xosé Lluis (2013): «Conciencia llingüística y testos asturianos medievales», en Lletres Asturianes 108: 87-106.

GIMÉNEZ, Gilberto (2009): «Cultura, identidad y memoria. Materiales para una sociología de los procesos culturales en las franjas fronterizas», en Frontera Norte, 21 (41). México. [Consulta a 3/10/2019, recuperáu de http://www.scielo.org.mx/scielo.php?script=sci_arttext\&pid=S0187$73722009000100001]$.

GonZÁlez RiaÑo, Xosé Antón (2002): Manual de Sociollingüística. Uviéu, ALLA.

GONZÁleZ RodrígueZ, Ruth y Ricardo SAAVEDRA FERnÁndEZ-CombarRo (2006): «Aproximación pragmática a la categorización de una lengua: la fala del Navia Eo», en T. Brandenberger y B. Schmid (eds.), Actas del VI Encuentro hispano suízo de filólogos noveles (Oviedo, 9 de mayo de 2006). Basel, Institut für Iberoromanistik.

GONZÁLEZ-QUEVEDO, Roberto (2019): «La normalización externa del asturiano», en Javier Giralt Latorre y Francho Nagore Lain (eds.), La normalización social de las lenguas minoritarias. Experiencias y procedimientos para la salvaguarda de un patrimonio inmaterial. Zaragoza, Universidad de Zaragoza: 58-81.

GuARDADO DIEZ, David (2008): Llingua estándar y normalización llingüística. La revitalización de les llingües subordinaes. Xixón, Araz Llibros.

LARROSA, Jorge (2019): Esperando no se sabe qué sobre el oficio de profesor. Barcelona, 
Candaya.

LóPez GiL, Mónica María y Félix Angulo Rasco (2015): «Sonorona o el rizoma de la cultura digital. Um estudio de caso», en Revista Portuguesa de Educação, 28 (1): 9-33.

LYON, David (2000): Posmodernidad. Madrid, Alianza Editorial. [2 ${ }^{\mathrm{a}}$ ed.].

LYOTARD, Jean-François (1991): La condición posmoderna. Informe sobre el saber. Madrid, Cátedra. [2a ed.].

MaIOZ BASTERRETXEA, Laura (2015): Pedagogía rizomática en la educación artística. (Trabayu de Fin de Máster). La Rioja, Universidad Internacional de La Rioja.

MARTín-BARBERO, Jesús (2002): «Desencuentros de la socialidad y reencantamientos de la identidad», n'Anàlisi, 29: 45-62.

MÉNDEZ, Benxamín y FREDO DE CARBEXE (2000): «Bases pra úa valorización sociollingüística en Asturias. El Navia-Eo», en Lletres Asturianes 75: 27-47.

PePPers, Don y Martha Rogers (1993): The One to One Future. New York, Currency Press Doubleday.

PITTA, Dennis A. (1998): «Marketing One-to-One and its dependence on Knolwledge discovery in databases», en Journal of consumer marketing, 15 (5): 468-480.

Rodríguez PÉREZ, Alma Silvia (2004): «La crisis de identidad y el conflicto lingüístico en la globalización», n'Actas del XV Congreso de la Asociación Internacional de Hispanistas. Vol. I. [Consulta a 7/10/2019, recuperáu de https://cvc.cervantes.es/literatura/aih/aih_xv.htm].

RoMAN ETXEBARrieTA, Gorka y Elizabeth PÉREZ-IZAGUIRRE (2018): «Identidades efímeras: análisis de estrategia de alianza etnolingüística en sistemas educativos multiculturales», n'Hizkuntzak, Ondarea eta Identitateak. Hezkuntza ikuspegia / Lengas, patrimòni e identitats. Perspectiva educativa. Lenguas, patrimonio e identidades. Perspectiva educativa / Langues, patrimoine et identités. Perspective éducative. Languages, Heritage and Identities. An Educational Perspective. Madrid, Delta Publicaciones: 315-322.

RuitiÑa TESTA, Cristóbal (2012): «La llingua asturiana na televisión autonómica. Presencia, audiencia y estándar», en Lletres Asturianes 106: 229-246.

- (2013): «Industrializar o perecer. Los medios de comunicación como motores de revitalización cultural», en Lletres Asturianes 108: 129-143

SÁNCHEZ ARRIETA, Antonio Jesús (2007): El valor del cliente como herramienta estratégica de gestión en un mercado industrial. (Tesis doctoral). Málaga, Universidad de Málaga.

Simmel, Georg (2010): Cultura líquida y dinero. Fragmentos simmelianos de la modernidad. Barcelona, Anthropos Editorial.

VERGARA L., Enrique (2006): «Medios de comunicación y globalización: ¿destrucción o reconstrucción de identidades culturales?», n'Anàlisi 33: 95-105.

VIEJO FERNÁNDEZ, Xulio (2017): «Reflexones marxinales sobro sociollingüística asturiana», en RFA 17: 201-223.

ZAPATA DUQUE, Jorge Fernando (2005): «Hipermedia y comunicación, un análisis a la luz del pensamiento rizomático», en Revista Virtual Universidad Católica del Norte, 14.

ZUFIAURRE, Benjamín (2007): ¿Se puede cambiar la educación sin contar con el profesorado? Reflexiones sobre treinta y cinco años de cambio en España: 1970-2005. Barcelona, Octaedro.

\subsection{Informes}

ALLA (2006): Informe sobre a fala ou gallego-asturiano. Úa perspectiva hestórica, social y llingüística. Uviéu, Academia de la Llingua Asturiana.

BRANDMANIC (2018): Estudio sobre Marketing de Influencers en España, 2018. [Consulta a 2/10/2019, recuperáu de https://www.brandmanic.com/ebooks/estudio-sobre-marketing-deinfluencers-en-espana-2018/].

DD.AA. (2018): Informe sobre la llingua asturiana 2018. Uviéu, Academia de la Llingua Asturiana. 
Grupo Banco Mundial (2019): «Población Total». Datos de libre acceso del Banco Mundial. Información en llinia. [Consulta a 2/09/2019, recuperáu de https://datos.bancomundial.org/].

Hevia Artime, Isabel (2018): Los jóvenes y la Lengua Asturiana. Avance de Resultados. Uviéu, Conseyería de la Mocedá del Principáu d'Asturies \& ALLA.

Llera RAmo, Francisco J. (2018): III Encuesta Sociolingüística de Asturias-2017. Avance de resultados. Uviéu, Academia de la Llingua Asturiana.

XDLA (2013): Informe sobre l'usu del asturianu na RTPA. Uviéu, XDLA.

\subsection{Llexislación}

Llei D'usu y PROMOCIÓN DEL BABLE/ASTURIANU, 1/98 de 23 de marzu. [Consulta a 11/10/2019, recuperáu de https://www.boe.es/buscar/doc.php?id=BOE-A-1998-10126].

\section{4. Webgrafía}

BARRANCo BARCElona, Justo (2017): «Pero, ¿qué es la modernidad líquida?», en La Vanguardia. [Consulta a 11/10/2019, recuperáu de https://www.lavanguardia.com/cultura/20170109/413213624617/modernidad-liquidazygmunt-bauman.html.].

BARRAYCOA, Javier (2017): «El multiculturalismo: una estrategia de dominación cultural y política (2)», en Posmodernia. [Consulta a 1/09/2019, recuperáu de http://www.posmodernia.com/el-multiculturalismo-una-estrategia-de-dominacion-culturaly-politica-y-2/.].

CORMIER, David (2014): «Rhizomatic learning - The community is the curriculum. P2PU». MOOC en llinia. [Consulta a 15/10/2019 https://courses.p2pu.org/en/courses/882/rhizomaticlearning-the-community-is-the-curriculum/].

Cuatrogotes Pproducciones S.L. (2018): [Consulta a 9/10/2019, www.cuatrogotes.com].

Llamedo Pandiella, Gonzalo (2019): «Silencios en-callados en la sociedad italiana», en Tonos Digital 36. [Consulta a 7/10/2019, recuperáu de http://www.tonosdigital.es/ojs/index.php/tonos/article/view/2117].

R[OJo], M[iguel] (2019a): «Ignacio Blanco, de Vox Asturias, se pone una montera picona en la Junta y critica que "la llingua es el objetivo del nacionalismo"», en La Nueva España. [Consulta a 24/10/2019, recuperáu de https://www.lne.es/asturias/2019/10/23/ignacioblanco-vox-asturias-pone/2547789.html].

- (2019b): «Entre los fines de la Academia de la Llingua no está la oficialidad del bable», n'El Comercio. [Consulta a 24/10/2019, recuperáu de https://www.elcomercio.es/culturas/actoconciliacion-academia-llingua-vox-20190326194042-nt.html]. 